\title{
Sharing Experience in the Application of Distributed Map of Intractable Epistaxis in Concealed Sites
}

\author{
Hui-Yi Deng ${ }^{1}$, Huijun Qiu ${ }^{1}$, Wei-Hao Wang ${ }^{1}$, and Qin-Tai Yang ${ }^{1}$ \\ ${ }^{1}$ Third Affiliated Hospital of Sun Yat-Sen University Department of Otorhinolaryngology \\ Head and Neck Surgery
}

May 21, 2020

\section{Sharing Experience in the Application ofDistributed Map of Intractable Epistaxis in Concealed Sites}

Huiyi. Deng ${ }^{1, \#}$, Huijun. Qiu ${ }^{1, \#}$, Weihao Wang ${ }^{1}$, Qintai. Yang ${ }^{1,2, *}$

1 Department of Otolaryngology-Head and Neck Surgery, The Third Affiliated Hospital, Sun Yat-sen University, Guangzhou, China

${ }^{2}$ Department of Allergy, The Third Affiliated Hospital, Sun Yat-sen University, Guangzhou, China

\# The authors contributed equally to this work.

* Corresponding author

Qintai Yang, MD, PhD

Department of Otolaryngology-Head and Neck Surgery

Department of Allergy

The Third Affiliated Hospital, Sun Yat-sen University

No. 600 Tianhe Road, Guangzhou 510630, China

Phone: 020-85252239

E-mail: yang.qt@163.com

\section{Funding}

This work was supported by the National Natural Science Foundation of China (Grant No. 81670912 and 81870704) and The Third Affiliated Hospital of Sun Yat-Sen University, Clinical Research Program (No. QHJH201901 and No. 2019006).

\section{Conflicts of interest}

The authors declare that they have no conflicts of interest. This work described was original research that has not been published previously, and not under Consideration for publication elsewhere.

\section{Key words}

Epistaxis; Big Data; Distributed Map; Nasal Endoscope;Bipolar Radiofrequency

To the Editor: 
Epistaxis is a frequently encountered otolaryngologic emergency, occurring in up to $60 \%$ of the general population, with one in ten of those affected seeking for medical attention. It accounts for one in 200 emergency department visits ${ }^{1}$. Here are a few classic cases in nearly a year in our hospital. We received and cured three classic cases referred by lower hospitals, who were elderly patients with recurrent epistaxis (Fig. 1). All the patients failed to stop bleeding after repeated anterior and posterior nostril packing and even interventional embolization of internal maxillary artery from external carotid artery, with a total cost of more than 50,000 yuan. At the first visit, the patients had anemic appearance and were suffering from pain, emotional anxiety due to anterior and posterior nostril packing. According to the "distributed map" we drew before, the bleeding point was quickly located in both patients, showing that the bleeding site was the second most common location of refractory epistaxis. Stopped bleeding via bipolar radiofrequency ablation under nasal endoscope without any stuffing. The treatment takes only 3 minutes and costs hundreds. The treatment is satisfying, cheap and time-saving. Here, it is difficult to pack sufficiently because of middle turbinate blocking. Additionally, it is supplied by the posterior ethmoidal artery, so the interventional embolization of the external carotid artery system is ineffective.

Previously, we searched the Chinese literature from 2009 to 2019 with the key words "intractable epistaxis, concealed epistaxis, refractory epistaxis and severe epistaxis". Besides, the same type of epistaxis patients in our hospital were analyzed. After classifying the most common concealed sites of epistaxis, we figured out that the frequency of bleeding areas came as follows: the vault of inferior nasal meatus in 3843 cases(31.58\%), the olfactory sulcus of middle turbinate in 3606 cases(29.63\%), the posterior regions of middle meatus in 1398 cases(11.49\%), the regions of deviation of nasal septum in 1094 cases(8.99\%), the foremost regions of nasal cavity in 775 cases $(6.37 \%)$, and the others or uncertain in 1453 cases(11.94\%) Finally, we drew a concise "distributed map" of epistaxis on the basis of the concealed bleeding areas and offending vessels (Fig.2).

In addition, we summarized the optimal diagnosis and treatment of refractory epistaxis. Firstly, maximum nasal contraction and topical anesthesia under nasal endoscope (1:1000 epinephrine combined with 1\% tetracaine cotton patch; pure epinephrine cotton patch if necessary). Then, sufficient clearance of bleeding with a suitable sucker. Sometimes the fracture displacement of the middle / inferior turbinate was required. Then, from the surface to the deep interior, we search the bleeding sites based on the "distributed map" in turn as follows: the foremost regions of nasal cavity, the regions of common nasal meatus and nasal septum; the posterior regions of middle meatus (medial turbinate fracture to inside), the olfactory sulcus area of nasal septum (middle turbinate fracture to outside) and the vault of inferior nasal meatus (inferior turbinate fracture to inside). Locate the bleeding site accurately and stop bleeding via bipolar radiofrequency ablation under nasal endoscope without nasal packing. Patients who were not clear were treated with interventional examination and embolization as appropriate, and were followed up for 1 to 3 months.

As far as I'm concerned, we think that some epistaxis is difficult to treat because it is unable to define the bleeding site and the traditional packing has an anatomical "blind spot". In this paper, we consider this kind of epistaxis as refractory epistaxis, which refers to a large amount of blood gushing out from the front nose or oropharynx, but routine anterior rhinoscopy or nasal endoscopy can't identify the bleeding site, and conventional nasal packing is often ineffective as well. The main responsible vessels are sphenopalatine artery, anterior and posterior ethmoidal artery. The key is to find the bleeding site accurately and stop the bleeding effectively. However, it is not easy. First of all, the middle turbinate, inferior turbinate and deviation of the nasal septum block the searching areas described above. Thus, it is difficult to identify the bleeding point without rich experience and illuminating system. In this study, the hidden bleeding sites and responsible blood vessels were drawn into a "distributed map" of epistaxis (Fig.2). It shows the two closely adjacent anatomical areas of the lateral wall of the nasal cavity and the nasal septum as well as the situation of occult epistaxis by the effect of turning pages like a book. We aim to take the picture as a guide to search for key bleeding sites, in order to improve management of epistaxis for young doctors and reduce blind nasal packing.

For epistaxis, nasal packing is still a common treatment in ENT emergency, especially in county hospitals. However, the traditional nasal packing not only brings great pain to patients, but also has poor hemostatic 
effect, as well as serious complications such as nasal mucosal injury, nasal septum perforation, nasal alar injury and so on ${ }^{5}$. Therefore, in accordance with Nikolaou, $\mathrm{G}^{6}$, we strongly suggest that in the era of nasal endoscope and Precision Medicine, we should optimize the mode of diagnosis and treatment of epistaxis, sticking to the principle of "from surface to deep, from simple to complex, from non-invasive to invasive", focusing on searching for bleeding sites in concealed areas based on the map. We search for bleeding sites under nasal endoscope and stop bleeding, so as to avoid nasal packing as far as possible and improve the quality of life of patients on the basis of improving the level of diagnosis and treatment.

\section{References:}

1. DJ P, YM C, MP M, JA E, AJ P, CA C. Epidemiology of epistaxis in US emergency departments, 1992 to 2001. Annals of emergency medicine 2005; 46:77-81.

2. Womack JP, Kropa J, Jimenez Stabile M. Epistaxis: Outpatient Management. American family physician 2018; 98:240-245.

3. Krulewitz NA, Fix ML. Epistaxis. Emergency medicine clinics of North America 2019; 37:29-39.

4. ZA K, GF P. Epistaxis: an overview. Emergency medicine clinics of North America 2013; 31:443-454.

5. Sireci F, Speciale R, Sorrentino R, Turri-Zanoni M, Nicolotti M, Canevari FR. Nasal packing in sphenopalatine artery bleeding: therapeutic or harmful? European archives of oto-rhino-laryngology : official journal of the European Federation of Oto-Rhino-Laryngological Societies (EUFOS) : affiliated with the German Society for Oto-Rhino-Laryngology - Head and Neck Surgery 2017; 274:1501-1505.

6. G N, D H, MB S. Discomfort and costs in epistaxis treatment. European archives of oto-rhino-laryngology : official journal of the European Federation of Oto-Rhino-Laryngological Societies (EUFOS) : affiliated with the German Society for Oto-Rhino-Laryngology - Head and Neck Surgery 2013; 270:2239-2244.

\section{Figure Legends}

Figure. 1: Pictures of nasal endoscope of epistaxis in two cases. (A/C/E. The left/right olfactory cleft is the bleeding point; B/D/F. Great Hemostasis effect by bipolar radiofrequency under nasal endoscope)

Figure. 2: The distributed map and relevant offending vessels of concealed epistaxis. 

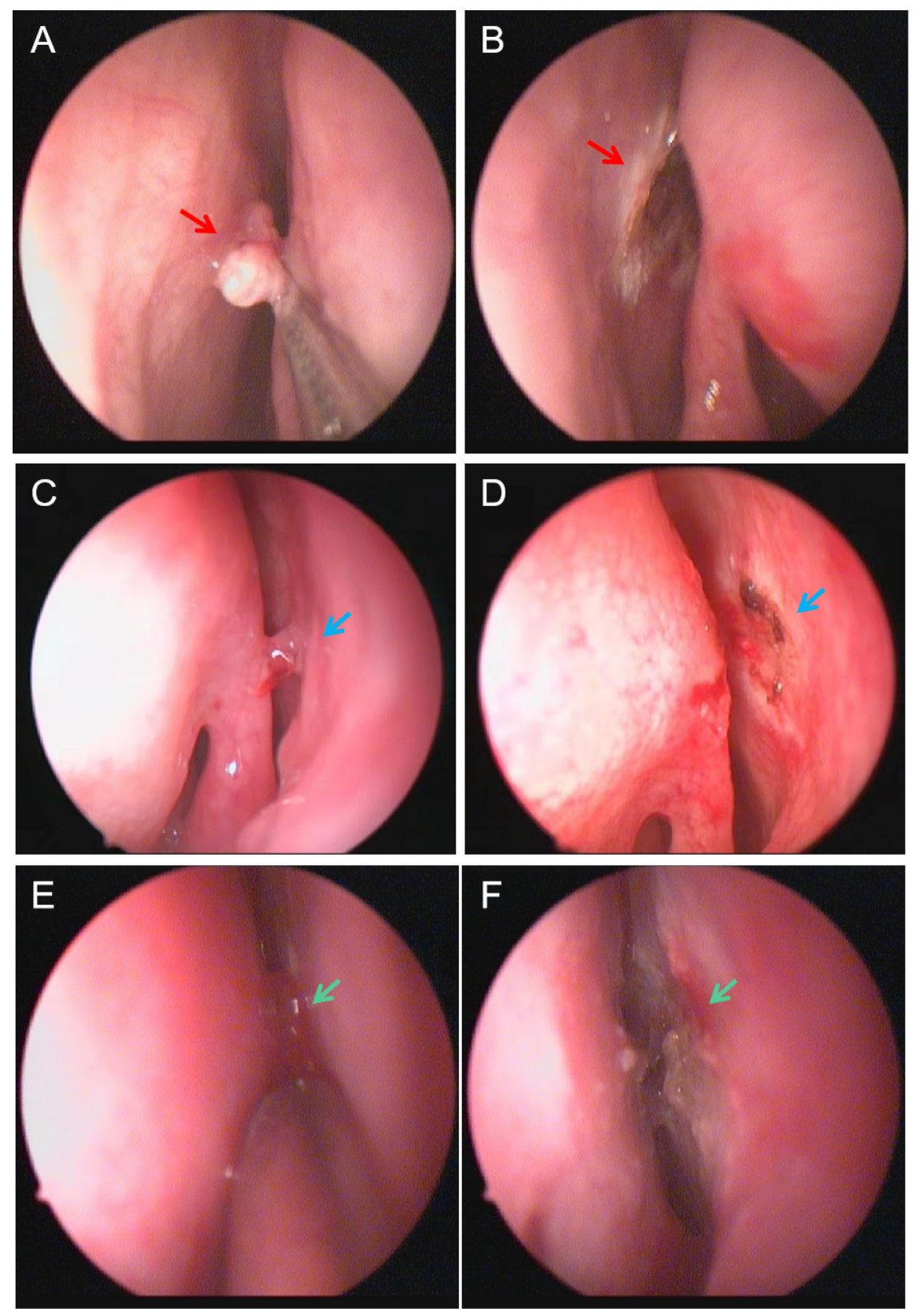


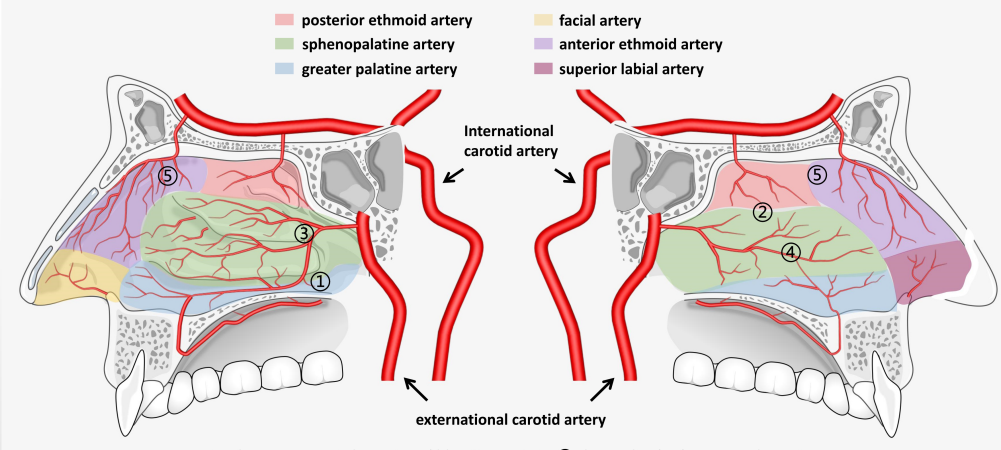

Numbers represent the order of bleeding points: (1) the vault of inferior nasal meatus

(2) the olfactory sulcus of middle turbinate (3) the posterior regions of middle meatus

(4) the regions of deviation of nasal septum (5) the foremost regions of nasal cavity 